\title{
Psicooncología
}

ISSN: 1696-7240

\section{Efectos del tipo de tratamiento y grupo de riesgo en la calidad de vida y la información en pacientes con cáncer de próstata}

\author{
Natalia Oraá-Tabernero'; Juan Antonio Cruzado Rodríguez ${ }^{2, *}$; Gustavo Ossola Lentati ${ }^{3}$ Natalia \\ Martínez del Pino ${ }^{4}$; Montserrat Sánchez-Fuertes ${ }^{5}$; Fernando Javier Martínez-Castellanos ${ }^{6}$
}

Recibido: 1 de junio de 2017 / Aceptado: 25 de agosto de 2017

Resumen. Objetivo: Comprobar las diferencias en la calidad de vida y la información de los pacientes con cáncer de próstata según el tratamiento y grupo de riesgo, y valorar su asociación con las variables sociodemográficas y clínicas. Metodo: entre 2015-2016, 176 hombres con cáncer de próstata seleccionados aleatoriamente, que habían recibido tratamientos locales, hormonales o combinados fueron evaluados mediante entrevista y los cuestionarios EORTC QLQ-C30, EORTC QLQ-PR25 y EORTC QLQ-INFO25. Resultados: La calidad de vida estaba más deteriorada en las escalas física, de rol, social y sexual en el grupo de riesgo avanzado y los tratados con hormonoterapia. La prostatectomía era la que producía más problemas urinarios, y los tratamientos combinados mayor sintomatología hormonal. Los tratados con radioterapia consideraban la información más útil y se sentían más informados sobre la enfermedad, pruebas médicas y tratamientos que los que recibían tratamiento hormonal y estaban más satisfechos que los intervenidos con prostatectomía. Los mayores o con estudios básicos recibieron menos información y la consideraron menos útil. Conclusiones: La calidad de vida es satisfactoria y la sintomatología baja. Los hombres en riesgo avanzado y/o los que reciben hormonoterapia presentan mayor déficit en la calidad de vida. La información es deficitaria, sobre todo en los hombres con problemas urinarios y con peor calidad de vida. Hay poca o ninguna información en las áreas "otros servicios", "lugares de cuidado" y "pautas de autocuidado". Es necesario evaluar y tratar a los hombres más vulnerables. Además de formar en información al personal sanitario.

Palabras clave: Cáncer de próstata; grupo de riesgo; tratamientos; EORTC QLQ-C30; EORTC QLQPR25; EORTC QLQ-INFO25.

1 Facultad de Psicología; Universidad Complutense de Madrid. Madrid.

E-mail: noraa@cop.es

2 Facultad de Psicología; Universidad Complutense de Madrid. Madrid.

E-mail: jacruzad@ucm.es

3 Servicio de Radioterapia Oncológica; Fundación Rioja Salud. Logroño (La Rioja).

E-mail: gossola@riojasalud.es

4 Servicio de Urología; Hospital San Pedro de La Rioja. Logroño (La Rioja).

E-mail: natalia.martinezdelpino@osakidtza.eus

5 Unidad de codificación Hospital San Pedro; Fundación Rioja Salud. Logroño (La Rioja).

E-mail: msanchezf@riojasalud.es

6 Servicio de Urología; Hospital San Pedro de La Rioja. Logroño (La Rioja).

E-mail: fmcastellanos@riojasalud.es

* Dirección de correspondencia: Juan Antonio Cruzado. Facultad de Psicología; Universidad Complutense de Madrid. Campous Somosaguas. 28223-Madrid. España. E-mail: jacruzad@ucm.es 


\title{
[en] Effects of type of treatment and risk group on quality of life and information in patients with prostate cancer
}

\begin{abstract}
Objective: to determine the differences in the quality of life and the information of the patients with prostate cancer according to the treatment and the risk group, and assess its association with sociodemographic and clinical variables. Method: Between 2015-2016, 176 men with prostate cancer, selected at random, who had received local treatments, hormonal or combined, were evaluated through interviews and the questionnaires EORTC QLQ-C30, EORTC QLQ-PR25 and EORTC QLQINFO25. Results: The quality of life was more damaged on the physical, role, social and sexual scales in the advanced risk group and those treated with hormone therapy. The prostatectomy was producing more urinary problems, and the combined treatments, more hormonal symptoms. Those treated with radiation therapy considered the information more useful and felt more informed about the disease, medical tests and treatments than those receiving hormonal treatment, and they were more satisfied than those treated with prostatectomy. The elderly or those with basic studies received less information and considered it was less useful. Conclusions: The quality of life is satisfactory and the symptomatology is low. Men at advanced risk and/or those who receive hormone therapy have a higher deficit in their quality of life. The information is deficient, especially in men with urinary problems and those with worse quality of life. There is little or no information in the "other services", "places of care" and "self-care guide". It is necessary to evaluate and treat the most vulnerable men. In addition, it would be desirable to introduce a system of training on information for medical professionals.
\end{abstract}

Keywords: Prostate cancer; risk group; treatment; EORTC QLQ-C30; EORTC QLQ-PR25; EORTC QLQ-INFO25.

Sumario. 1. Introducción 2. Material y método 3. Análisis de datos 4. Resultados 5. Discusión 6. Agradecimientos 7. Referencias bibliográficas.

Cómo citar: Oraá-Tabernero N, Cruzado Rodríguez JA, Ossola Lentati G, Martínez del Pino N, SánchezFuertes M, Javier Martínez-Castellanos F. Efectos del tipo de tratamiento y grupo de riesgo en la calidad de vida y la información en pacientes con cáncer de próstata. Psicooncología 2017;14:241-54. Doi: 10.5209/PSIC.57083.

\section{Introducción}

En España, el cáncer de próstata es el tumor maligno con más incidencia, con una alta prevalencia y supervivencia a los 5 años, y baja mortalidad ${ }^{(1)}$.

Hoy en día se puede decidir entre diferentes opciones de tratamiento, en función de parámetros como el grupo de riesgo, que indica la probabilidad de que el cáncer de próstata crezca y se disemine ${ }^{(2)}$. En cuanto a los tratamientos, si el tumor está localizado en la próstata se puede optar por la prostatectomía radical o radioterapia radical externa o interna. Si la enfermedad está localmente avanzada o existen altas posibilidades de recaída se suele optar por tratamientos hormonales neoadyuvantes asociados a los locales. Y, si la enfermedad está avanzada la opción inicial más frecuente es el tratamiento hormonal ${ }^{(3)}$. Estos tratamientos aumentan la supervivencia pero producen efectos que a menudo deterioran la calidad de vida ${ }^{(4,5)}$. En este sentido, los estudios indican que es importante evaluarla en cualquier fase de la enfermedad debido a los efectos adversos de los tratamientos o la enfermedad, siendo los hombres de grupo de riesgo avanzado los más afectados ${ }^{(6-8)}$. Así, hoy en día hay un especial interés no sólo en valorar la eficacia de los tratamientos, sino también en conocer como la información trasmitida puede influir en la adaptación 
o en la propia calidad de vida. De hecho, una información centrada en cubrir las necesidades individuales, permite al paciente participar en los procesos de decisión, facilita la adaptación, aumenta la satisfacción y la calidad de vida ${ }^{(9,10)}$.

Este estudio pretende comprobar las diferencias del tipo de tratamiento y el grupo de riesgo en: a) la calidad de vida (EORTC QLQ-C30 y PR25), b) el nivel de información (EORTC QLQ-INFO25), además de valorar la asociación de las variables sociodemográficas (edad, niveles de estudio, tener pareja) y las variables clínicas (fase de tratamiento y de supervivencia) con la calidad de vida y la información recibida, y de estas dos últimas variables entre sí.

\section{Material y método}

\subsection{Muestra}

Se evaluaron a 176 pacientes $(81,48 \%)$ atendidos en el Servicio de Urología del Hospital San Pedro de Logroño y/o el Servicio de Radioterapia Oncológica de Fundación Rioja Salud diagnosticados de cáncer de próstata entre 2013-2015, y previamente 40 $(18,51 \%)$ fueron excluidos por motivos como rehusar a colaborar $(n=23)$ o no cumplir los criterios de selección $(\mathrm{n}=17)$. Se encontraban en tratamiento activo hormonal desde hacía un mínimo de 3 meses o en revisión como mínimo de 3 meses tras el tratamiento quirúrgico o radioterápico. Fueron excluidos aquellos que obtenían una puntuación de Karnofsky $\leq 80$ o Mini Mental $\leq 26$ puntos así como los que no entendieran castellano, fueran paliativos o tuvieran antecedentes de trastornos mentales.

\subsection{Variables e instrumentos}

Los pacientes se dividieron, en 4 grupos según el tipo de tratamiento: prostatectomía, radioterapia radical externa o interna, tratamiento combinado $u$ hormonal. Por otro lado, respecto al grupo de riesgo se consideraron 4 grupos de riesgo en función de la extensión del tumor, el valor PSA y la escala de Gleason (bajo, intermedio, alto y avanzado).

Como instrumentos de selección se utilizaron la Escala Karnofsky que mide la capacidad funcional ${ }^{(11)}$, y el Mini Examen Cognoscitivo que comprueba el deterioro $\operatorname{cognitivo}^{(12,13)}$.

Para evaluar la calidad de vida se utilizó el cuestionario general EORTC QLQ-C30 y el específico de cáncer de próstata EORTC QLQ-PR25 $5^{(14,15)}$. Ambos cuestionarios han demostrado ser válidos y fiables para muestras tanto internacionales como españolas $^{(16-19)}$.

Para evaluar la percepción y satisfacción de la información recibida durante la enfermedad o el tratamiento se utilizó el módulo EORTC QLQ-INFO25. Es un cuestionario con una buena consistencia interna y buena fiabilidad test-retest (correlación intraclase $>0,7)^{(20,21)}$.

Todas las puntuaciones de los cuestionarios de la EORTC se transforman en una puntuación lineal en escala de 0-100 de acuerdo con el manual de la EORTC ${ }^{(15)}$.

La descripción específica de los cuestionarios EORTC QLQ-C30; EORTC QLQPR25 y EORTC QLQ-INFO25 se detallan en la tabla 1. 
Tabla 1. Descripción de los cuestionarios de calidad de vida general y específica de cáncer de próstata (EORTC QLQ-C30 y PR25) e información recibida (EORTC QLQ-INFO25).

\begin{tabular}{|c|c|c|c|}
\hline $\begin{array}{c}\text { NOMBRE } \\
\text { INSTRUMENTO }\end{array}$ & EORTC QLQ-C30 & EORTC QLQ-PR25 & EORTC QLQ-INFO25 \\
\hline OBJETIVO & $\begin{array}{l}\text { Calidad de vida } \\
\text { general en oncología }\end{array}$ & $\begin{array}{l}\text { Calidad de vida en } \\
\text { cáncer de próstata }\end{array}$ & $\begin{array}{l}\text { Percepción y satisfacción } \\
\text { de la información recibida }\end{array}$ \\
\hline $\mathrm{N}^{0}$ ÍTEMS & 30 & 25 & 25 \\
\hline ESTRUCTURA & $\begin{array}{l}1 \text { escala global de } \\
\text { salud } \\
5 \text { escalas funcionales: } \\
\text { física, de rol, social, } \\
\text { emocional, cognitiva } \\
\text { y social } \\
3 \text { escalas de } \\
\text { síntomas: fatiga, } \\
\text { naúseas/vómitos, } \\
\text { dolor } \\
6 \text { ítems: disnea, } \\
\text { apetito, insomnio, } \\
\text { estreñimiento, } \\
\text { diarrea y dificultades } \\
\text { financieras }\end{array}$ & $\begin{array}{l}4 \text { escalas síntomas: } \\
\text { urinaria, intestinal, } \\
\text { hormonal y actividad } \\
\text { sexual } \\
1 \text { escala condicional } \\
\text { (uso prenda urinaria) } \\
1 \text { escala condicional } \\
\text { (funcionamiento } \\
\text { sexual) }\end{array}$ & $\begin{array}{l}4 \text { escalas (sobre } \\
\text { enfermedad, pruebas } \\
\text { médicas, tratamientos y } \\
\text { otros servicios) } \\
4 \text { ítems individuales } \\
\text { (lugares de cuidado, cosas } \\
\text { para bienestar, } \\
\text { satisfacción y utilidad) } \\
4 \text { ítems cualitativos } \\
\text { (información escrita, en } \\
\text { cd, y deseo de recibir más } \\
\text { o menos información) }\end{array}$ \\
\hline $\begin{array}{l}\text { TIEMPO } \\
\text { APLICACIÓN }\end{array}$ & 10-15 minutos & 10-15 minutos & 10-15 minutos \\
\hline $\begin{array}{l}\text { MODALIDAD DE } \\
\text { RESPUESTA }\end{array}$ & $\begin{array}{l}\text { Tipo Likert } 4 \\
\text { opciones de } \\
\text { respuesta, excepto } \\
\text { escala global de } \\
\text { salud con } 7 \\
\end{array}$ & $\begin{array}{l}\text { Tipo Likert } 4 \\
\text { opciones de respuesta }\end{array}$ & $\begin{array}{l}\text { Tipo Likert } 4 \text { opciones de } \\
\text { respuesta, excepto ítems } \\
20,21,23 \text { y } 24 \text { cualitativos } \\
\text { (si/no) }\end{array}$ \\
\hline INTERPRETACIÓN & $\begin{array}{l}\text { A más puntuación } \\
\text { más calidad de } \\
\text { vida pero más } \\
\text { sintomatología }\end{array}$ & $\begin{array}{l}\text { A más puntuación } \\
\text { más sintomatología y } \\
\text { mejor funcionamiento } \\
\text { sexual }\end{array}$ & $\begin{array}{l}\text { A más puntuación } \\
\text { mayor información, más } \\
\text { necesidad de recibir más } \\
\text { información y mayor } \\
\text { satisfacción }\end{array}$ \\
\hline
\end{tabular}

\subsection{Procedimiento}

Antes de comenzar la entrevista, se informaba a los sujetos de los objetivos y características de la investigación y firmaban el consentimiento informado. Posteriormente se recogían los datos sociodemográficos y se aplicaban los cuestionarios EORTC QLQ-C30, EORTC QLQ-PR25 y el EORTC QLQ-INFO25.

El Comité Ético dictaminó que el estudio cumplía los aspectos metodológicos, éticos y legales que permitían su realización.

\section{Análisis de datos}

Se obtuvieron los datos descriptivos de la muestra: frecuencias de las variables sociodemográficas y clínicas, las medias y desviaciones típicas de las variables cuantitativas. 
Para comprobar las diferencias según el tratamiento y el grupo de riesgo en la calidad de vida (EORTC QLQ-C30 y PR25) y en la información (EORTC QLQINFO25), se llevó a cabo la prueba de Kruskall Wallis con prueba post hoc de contraste Dunn-Bonferroni y se calculó el tamaño del efecto mediante la d de Cohen.

Se calculó la correlación de Pearson entre la calidad de vida, el nivel de información y la edad, para comprobar las posibles diferencias significativas en la calidad de vida y la información en función de tener pareja se llevó a cabo la prueba t de Student y para el nivel de estudios y fase de supervivencia la prueba de Kruskall Wallis.

El análisis de datos se realizó con el paquete estadístico SPSS v 22. Un valor asociado $\mathrm{p}<0,05$ fue considerado como estadísticamente significativo.

\section{Resultados}

La mayoría de los sujetos tenían edades superiores a 60 años, con estudios básicos, jubilados y con pareja, diagnosticados con grupo de riesgo bajo y en seguimiento de 24-36 meses. Los tratamientos más frecuentes fueron las técnicas radioterápicas y el tratamiento combinado (tabla 2).

Tabla 2. Características demográficas y clínicas de los pacientes.

\begin{tabular}{|c|c|c|}
\hline VARIABLES & & \\
\hline \multirow{2}{*}{ EDAD } & \multicolumn{2}{|l|}{$68,32(7,58)^{\mathrm{a}}$} \\
\hline & $N$ & $\%$ \\
\hline \multicolumn{3}{|l|}{ NIVEL ACADÉMICO } \\
\hline Básicos & 110 & 62,5 \\
\hline Secundarios & 41 & 23,3 \\
\hline Superiores & 25 & 14,2 \\
\hline \multicolumn{3}{|l|}{ SITUACIÓN LABORAL } \\
\hline Jubilado & 134 & 76,1 \\
\hline En activo & 31 & 17,6 \\
\hline Baja Laboral & 11 & 6,3 \\
\hline \multicolumn{3}{|l|}{ PAREJA } \\
\hline Con pareja & 144 & 81,8 \\
\hline Sin pareja & 35 & 18,2 \\
\hline \multicolumn{3}{|l|}{ GRUPO DE RIESGO } \\
\hline Bajo & 70 & 39,8 \\
\hline Intermedio & 53 & 30,1 \\
\hline Alto & 45 & 25,6 \\
\hline Avanzado & 8 & 4,5 \\
\hline \multicolumn{3}{|c|}{ TIPO DE TRATAMIENTO } \\
\hline Prostatectomia & 34 & 19,3 \\
\hline Técnicas Radioterápicas & 51 & 29,0 \\
\hline Tratamiento Combinado & 64 & 36,4 \\
\hline Tratamiento Hormonal & 27 & 15,3 \\
\hline
\end{tabular}




\begin{tabular}{lll}
\hline FASE SUPERVIVENCIA & & \\
$3-12$ meses & 47 & 26,7 \\
$12-24$ meses & 47 & 26,7 \\
$24-36$ meses & 82 & 46,6 \\
\hline
\end{tabular}

a Media (Desviación típica).

En la tabla 3 se exponen los datos para la muestra total en calidad de vida. Los resultados de la muestra total indican que las puntuaciones en las escalas funcionales son satisfactorias, siendo algo más baja la escala emocional. La sintomatología general y específica del cáncer de próstata es baja, siendo las más afectadas el insomnio, la actividad sexual y los síntomas urinarios.

Tabla 3. Media y Desviaciones Típicas de los Cuestionarios EORTC QLQ-C30, PR25 e INFO25

\begin{tabular}{lccc}
\hline \multicolumn{1}{c}{ EORTC QLQ-C30 } & Media & $\begin{array}{c}\text { Desviación } \\
\text { Típica }\end{array}$ & $\begin{array}{c}\text { Intervalo de confianza } \\
\text { para la media (95\%) }\end{array}$ \\
\hline Escala global de salud & 76,75 & 17,26 & $74,18-79,32$ \\
\hline Escalas Funcionales & & & \\
Funcionamiento físico & 88,44 & 18,68 & $85,66-91,22$ \\
Funcionamiento de rol & 86,55 & 28,26 & $82,34-90,75$ \\
Funcionamiento emocional & 77,74 & 25,10 & $74,01-81,48$ \\
Funcionamiento cognitivo & 80,58 & 26,85 & $76,59-84,58$ \\
Funcionamiento social & 89,96 & 21,53 & $86,75-93,16$ \\
\hline Escala síntomas & & & \\
Fatiga & 14,45 & 22,42 & $11,12-17,79$ \\
Nauseas & 1,42 & 7,31 & $0,33-2,50$ \\
Dolor & 9,65 & 18,20 & $6,95-12,36$ \\
Disnea & 5,68 & 16,11 & $3,28-8,07$ \\
Insomnio & 23,67 & 33,82 & $18,64-28,70$ \\
Pérdida de apetito & 5,11 & 16,87 & $2,60-7,62$ \\
Estreñimiento & 15,15 & 28,46 & $10,91-19,38$ \\
Diarrea & 10,60 & 24,19 & $7,00-14,20$ \\
Dificultades financieras & 1,89 & 10,52 & $0,32-3,45$ \\
\hline & \multirow{2}{c}{ EORTC QLQ-PR25 } & Desviación & Intervalo de confianza \\
para la media (95\%)
\end{tabular}




\begin{tabular}{lccc}
\hline EORTC QLQ-INFO25 & Media & $\begin{array}{c}\text { Desviación } \\
\text { Típica }\end{array}$ & $\begin{array}{c}\text { Intervalo de confianza } \\
\text { para la media (95\%) }\end{array}$ \\
\hline Puntuación global & 43,03 & 9,69 & $41,59-44,47$ \\
\hline Escalas & & & \\
Información sobre enfermedad & 53,88 & 23,93 & $50,32-57,44$ \\
Información sobre pruebas médicas & 66,41 & 26,71 & $62,43-70,38$ \\
Información sobre tratamientos & 61,96 & 27,92 & $57,80-66,11$ \\
Información sobre otros servicios & 7,67 & 12,48 & $5,81-9,52$ \\
\hline Ítems individuales & & & \\
Información sobre lugares de cuidado & 3,97 & 14,36 & $1,84-6,11$ \\
Información pautas autocuidado & 13,25 & 23,11 & $9,81-16,69$ \\
Información escrita & 55,68 & 49,81 & $48,27-63,09$ \\
Información cd & 1,13 & 10,62 & $-0,44-2,71$ \\
Satisfacción información & 73,86 & 27,34 & $66,79-77,93$ \\
Recibir más información & 17,61 & 38,20 & $11,93-23,29$ \\
Recibir menos información & 1,70 & 12,98 & $-0,22-3,63$ \\
Utilidad de la información & 72,91 & 28,15 & $68,72-77,10$ \\
\hline
\end{tabular}

Los resultados de la tabla 4 muestran diferencias en la calidad de vida según el tratamiento. Así, los pacientes que reciben hormonoterapia tienen significativamente peor funcionamiento físico que los que reciben prostatectomía o radioterapia, peor funcionamiento social respecto a las terapias radioterápicas y combinada y los que más deteriorados tienen el funcionamiento de rol. Por otro lado, la intervención de prostatectomía produce significativamente más dificultades urinarias que la radioterapia, y el tratamiento combinado el que más cambios hormonales, principalmente con respecto a la prostatectomía y radioterapia. Para terminar, los pacientes intervenidos con prostatectomía o radioterapia presentan significativamente mejor actividad sexual que los tratados con hormonas o combinados (tabla 4).

En la tabla 5 se exponen los resultados de la calidad de vida en función del grupo de riesgo, las personas que se encuentran en una situación avanzada son las que presentan significativamente peor estado de salud, funcionamiento físico, de rol, social y sexual, además de más fatiga y dolor.

En relación a la información, la puntuación global y la de las escalas del EORTC QLQ-INFO25 son medias-bajas a excepción de la "información sobre otros servicios" y los ítems "otros lugares de cuidado" e "información en CD" que son muy bajos. El resto de los ítems individuales son bajos, y en la satisfacción y utilidad de la información se obtienen puntuaciones satisfactorias (tabla 3).

Por otro lado, hay diferencias significativas en la información en función del tratamiento. Los pacientes que son tratados con técnicas radioterápicas se sienten significativamente más informados sobre la enfermedad, pruebas médicas y tratamientos que los que reciben hormonoterapia además de considerarla más útil. Además, son los que reciben radioterapia los más satisfechos con la información recibida sobre todo con respecto a los intervenidos con prostatectomía. No existen diferencias en la información recibida en función del grupo de riesgo (tabla 6). 


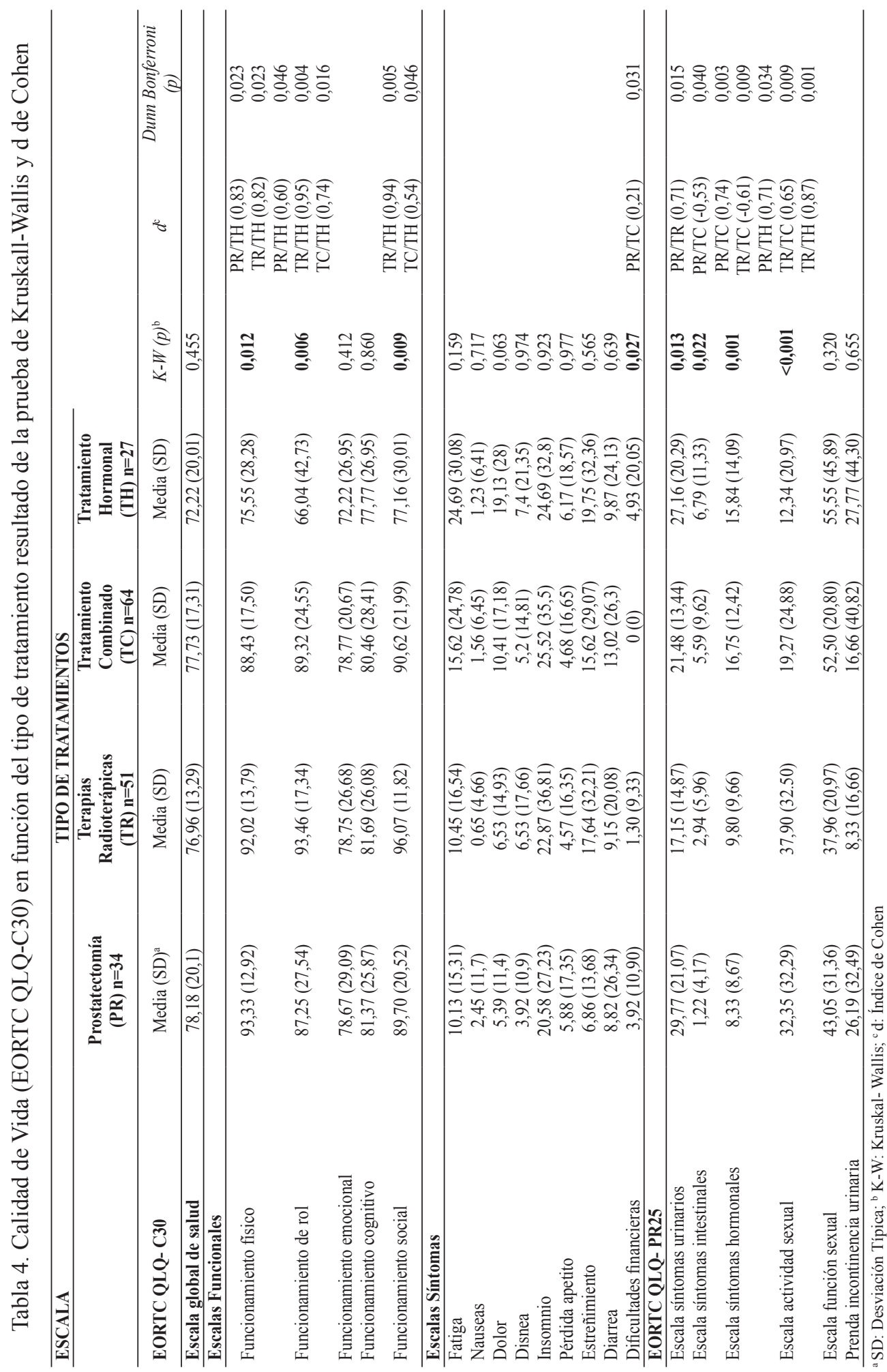




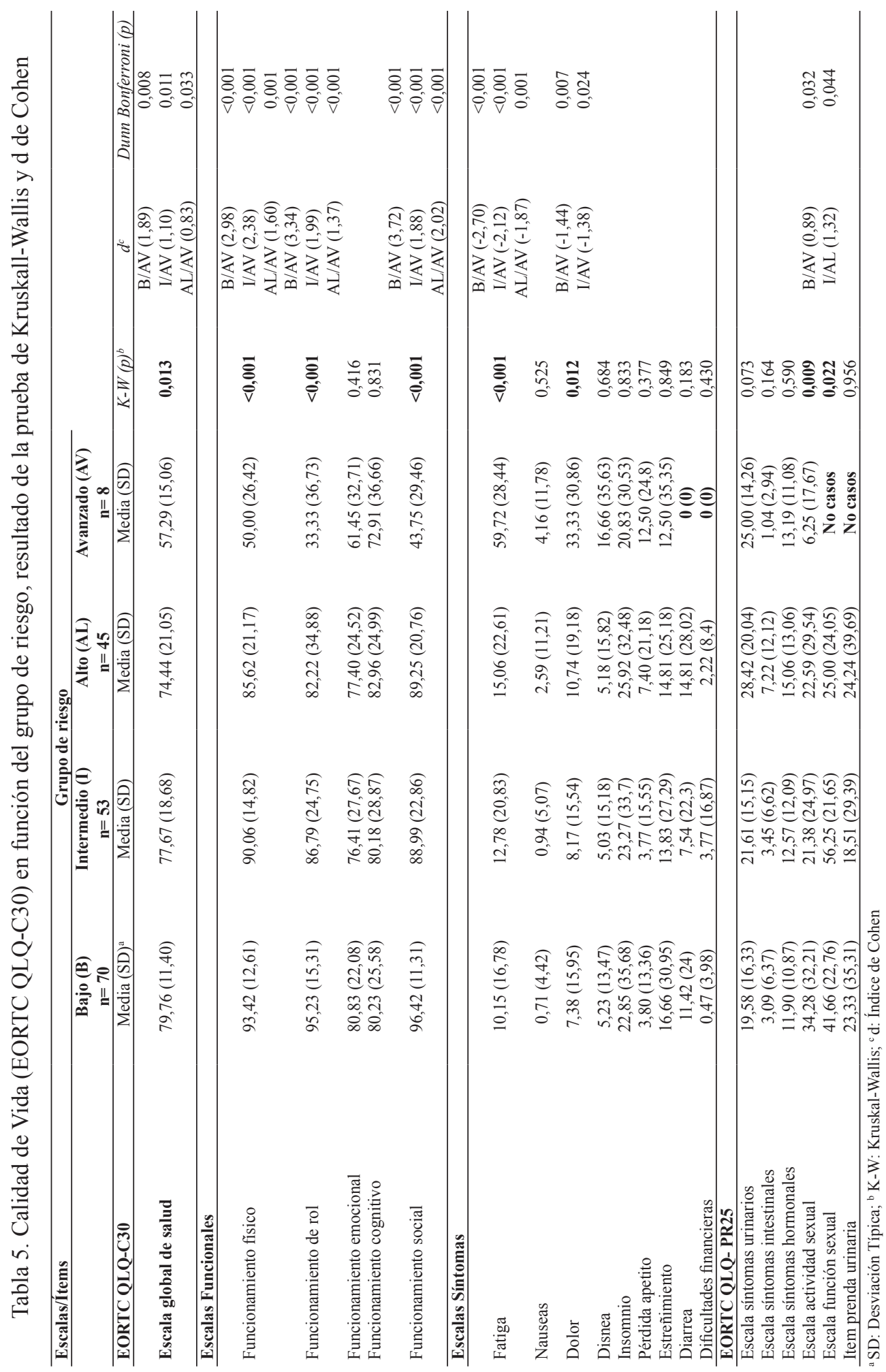


(2)

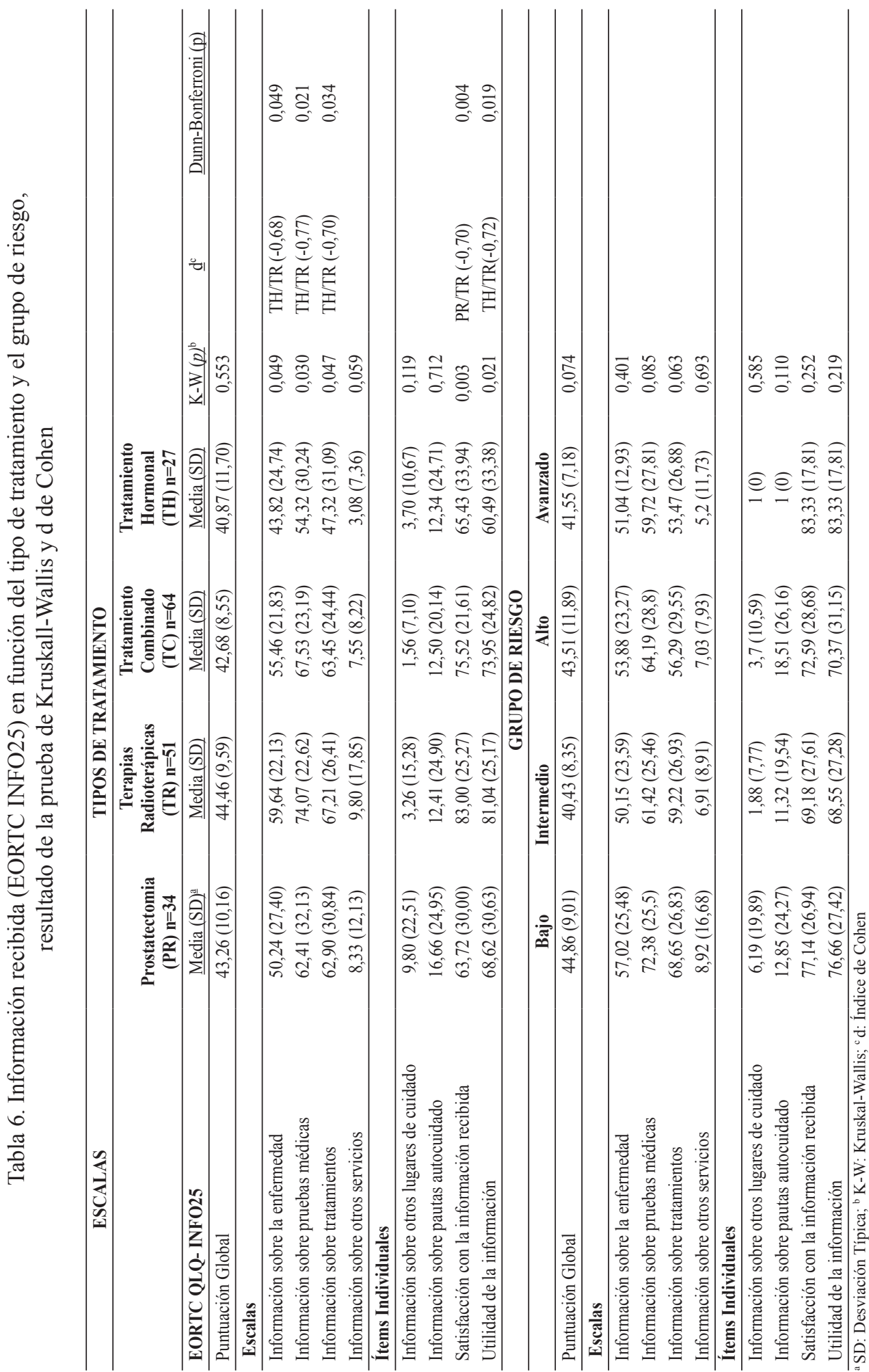


Por otro lado, con respecto a las variables sociodemográficas asociadas a la calidad de vida o la información, la edad no influye de manera significativa en la calidad de vida, salvo en la actividad sexual $(\mathrm{r}=-0,44 ; \mathrm{p}<0,01)$. Además, los hombres mayores reciben menos información sobre la enfermedad $(\mathrm{r}=-0,24 ; \mathrm{p}=0,001)$, pruebas médicas $(\mathrm{r}=-0,19 ; \mathrm{p}=0,014)$, tratamiento $(\mathrm{r}=-0,27 ; \mathrm{p}<0,001)$ y la consideran menos útil $(\mathrm{r}=-0,16 ; \mathrm{p}=0,037)$, y los que tienen estudios básicos reciben menos información sobre tratamientos ( $d$ de Cohen $=-0,64 ; \mathrm{p}<0,001)$.

Con el resto de las variables sociodemográficas o clínicas no existen diferencias en la calidad de vida o la información.

Las correlaciones de Pearson entre la calidad de vida y la información son bajas. Los hombres que se sienten más satisfechos con la información tienen mejor funcionamiento físico $(\mathrm{r}=0,18 ; \mathrm{p}=0,018)$, de rol $(\mathrm{r}=0,15 ; \mathrm{p}=0,046)$, emocional $(\mathrm{r}=0,2$; $\mathrm{p}=0,008)$ y social $(\mathrm{r}=0,24 ; \mathrm{p}=0,002)$ y menos síntomas urinarios $(\mathrm{r}=-0,32 ; \mathrm{p}<0,001)$, intestinales $(r=-0,24 ; p=0,002)$ y hormonales $(r=-0,21 ; p=0,005)$.

Además, los tratamientos hormonales son más utilizados en personas mayores en comparación con la prostatectomía o las técnicas radioterápicas ( $\mathrm{d}$ de Cohen $=-0,93$; $\mathrm{p}<0,001 \mathrm{y} \mathrm{d}$ de Cohen $=0,87 ; \mathrm{p}<0,001)$, y no existen diferencias en función del nivel de estudios ni tener pareja.

Existe una asociación significativa entre el grupo de riesgo y el tipo de tratamiento (Chi cuadrado=91,137; $\mathrm{p}<0,01$ ), el grupo de riesgo bajo recibe más técnicas radioterápicas $(57,1 \%)$, el intermedio y alto terapias combinadas $(64,2 \%$ y $44,4 \%$ respectivamente), y los avanzados terapias hormonales (75\%).

\section{Discusión}

La existencia de diferentes tratamientos, la alta incidencia y baja mortalidad, influyen en que el facultativo se interese por conocer cómo los tratamientos afectan a la calidad de vida de sus pacientes, qué factores pueden influir en la adaptación y, de qué herramientas disponen para afrontar las dificultades.

Los pacientes de nuestro estudio tienen una calidad de vida global media, con una funcionalidad adecuada y una sintomatología baja. Estos resultados coinciden en su mayoría con los datos de referencia del EORTC QLQ-C30 que indican una calidad de vida media, funcionalidad satisfactoria, y sintomatología baja ${ }^{(15)}$. También son similares a los obtenidos en la validación del EORTC QLQ-PR25, en los que existe una sintomatología moderada, siendo el área sexual y urinaria las más afectadas ${ }^{(19)}$, aunque diferenciándonos de la validación española en que nuestra población puntúa más alto en actividad sexual y menos en funcionamiento sexual ${ }^{(17)}$.

La calidad de vida de nuestros pacientes varía con respecto a los tratamientos recibidos. Así, los pacientes con tratamientos hormonales son los que más afectada tienen su calidad de vida en el funcionamiento físico, de rol y social. Fossa et al., concluyeron que los tratamientos, sobre todo los hormonales, reducen la calidad de vida y producen efectos adversos (principalmente en el área sexual) ${ }^{(22)}$. En nuestros pacientes, la prostatectomía es la que más afecta al área urinaria y, el tratamiento combinado al área hormonal. En cuanto al área de actividad sexual, el tratamiento combinado y el hormonal son los que más la deterioran. Estos resultados coinciden con estudios anteriores que indican que tras la prostatectomía aparece una mayor sintomatología urinaria, y que en general el uso de hormonas deteriora el área sexual ${ }^{(22,23)}$. 
Los pacientes del grupo de riesgo avanzado manifiestan tener peor funcionamiento físico, de rol y social que el resto de los grupos. Además, estos pacientes consideran su salud general más baja que los demás. Vanagas et al., obtuvieron resultados similares y concluyeron que los pacientes con estadios IV (grupo de riesgo avanzado) puntuaban peor en la calidad de vida, en las áreas de funcionamiento emocional y social ${ }^{(24)}$.

Una información adecuada puede influir en que el paciente se adapte mejor a los cambios. El nivel de información fue bajo, semejante a los encontrados en muestras internacionales y española ${ }^{(20,21)}$. Así, la puntuación global del test en ambos es baja $\mathrm{y}$, las puntuaciones de las escalas sobre enfermedad, pruebas médicas y tratamiento son medias, siendo algo superior en los estudios de referencia a excepción de la escala "información sobre los tratamientos". En ambos estudios las escalas "otros servicios" y "lugares de cuidado" son muy bajas, y la satisfacción y la utilidad de la información aceptable. Investigaciones recientes han concluido que existe un porcentaje elevado de no satisfacción y de carencias en la información ${ }^{(25,26)}$. Por otro lado, si comparamos la satisfacción de la información en función de los tratamientos, los pacientes que reciben radioterapia son los que se sienten más satisfechos. Tal como indicaba Majumder et al., esta diferencia puede deberse al hecho de que los pacientes que reciben radioterapia son atendidos tanto por el urólogo como por el especialista en radioterapia por lo que tienen más oportunidades de recibir más información ${ }^{(25)}$.

La debilidad de las asociaciones entre la información y la calidad de vida de nuestro estudio puede deberse a las puntuaciones tan bajas en las escalas de la información. A pesar de esto, coincidimos con otros resultados en que las personas más satisfechas con la información puntúan mejor en las áreas de calidad de vida y, presentan menos sintomatología ${ }^{(9,10,25,27)}$. Tal como indicaba Burt et al., el facultativo debe facilitar la información necesaria, teniendo en cuenta las características de cada paciente, con información escrita o digital para minimizar el impacto de la enfermedad y los tratamientos ${ }^{(28)}$.

En conclusión, los hombres en grupo de riesgo avanzado, como es de esperar, tienen peor calidad de vida, y en nuestro caso en las escalas global de salud, funcionamiento físico, de rol y social, y presentan más fatiga y dolor. Los hombres con tratamientos hormonales son los que presentan peor calidad de vida principalmente en el funcionamiento físico, de rol y social, y en la actividad sexual. La prostatectomía es la que más deteriora el área urinaria y los tratamientos combinados los que más síntomas hormonales producen. En cuanto a la información debe mejorar porque es deficitaria. Los hombres que necesitan más información son los que padecen más problemas urinarios o presentan peor calidad de vida. La mayoría de los pacientes no reciben información acerca de "otros servicios", "lugares de cuidado", y "pautas de autocuidado".

Es necesario llevar a cabo tratamientos inter y multidisciplinares que aseguren una atención integral en estos pacientes.

\section{Agradecimientos}

Agradecer al Dr. Enrique Ramalle-Gomara y la Dra. Josefa Gil-de Gómez Barragan su ayuda y colaboración con la metodología y análisis de esta investigación, y a la Dra. Elena Domínguez-Garrido su ayuda en la revisión de este artículo. 


\section{Referencias bibliográficas}

1. Ferlay J, Soerjomataram I, Dikshit R, Eser S, Mathers C, Rebelo M, et al. Cancer incidence and mortality worldwide: sources, methods and major patterns in GLOBOCAN 2012. Int J Cancer 2015; 136: E359-386. doi: 10.1002/ijc.29210

2. Heidenreich A, Bellmunt J, Bolla M, Joniau S, Mason M, Matveev V, et al. EAU guidelines on prostate cancer. Part 1: screening, diagnosis, and treatment of clinically localised disease. Eur Urol 2011; 59): 61-71. doi: 10.1016/j.eururo.2010.10.039

3. SEOM: Sociedad Española Oncología Médica [Internet]. Madrid: SEOM; 2015 [actualizado 30 Jun 2015 [Acceso 27 sept 2016]. Cáncer de próstata [aprox. 1 pantalla]. Disponible en: http://www.seom.org/es/informacion-sobre-el-cancer/info-tipos-cancer/ genitourinario/prostata?start=10

4. Serdà B-C, del Valle A, Marcos R, Monreal P. Beneficios de un programa de ejercicio de fuerza para la mejora de la calidad de vida del hombre con cáncer de próstata. Psicooncología 2009; 6: 211-26.

5. Serdà B-C, del Valle A. Cáncer de próstata y calidad de vida: análisis del cambio en la respuesta a partir de una triangulación entre métodos. Psicooncología 2012; 9: 439-51. doi: 10.5209/rev_PSIC.2013.v9.n2-3.40914

6. Eton DT, Lepore SJ. Prostate cancer and health-related quality of life: a review of the literature. Psychooncology 2002; 11: 307-26. Doi: 10.1002/pon.572

7. Couper JW, Love AW, Dunai JV, Duchesne GM, Bloch S, Costello AJ, et al. The psychological aftermath of prostate cancer treatment choices: a comparison of depression, anxiety and quality of life outcomes over the 12 months following diagnosis. Med J Aust 2009; 190( Suppl): S86-89.

8. Vanagas G, Mickeviciene A, Ulys A. Does quality of life of prostate cancer patients differ by stage and treatment? Scand J Public Health 2013; 41: 58-64. doi: $10.1177 / 1403494812467503$

9. Lamers RED, Cuypers M, Husson O, de Vries M, Kil PJM, Ruud Bosch JLH, et al. Patients are dissatisfied with information provision: perceived information provision and quality of life in prostate cancer patients. Psychooncology 2016; 25: 633-40. doi: 10.1002/pon.3981.

10. Nicolaisen M, Müller S, Patel HRH, Hanssen TA. Quality of life and satisfaction with information after radical prostatectomy, radical external beam radiotherapy and postoperative radiotherapy: a long-term follow-up study. Clin J Oncol Nurs 2014; 23: 3403-14. doi: 10.1111/jocn.12586.

11. Karnofsky DA, Abelmann WH, Craver LF, Burchenal JH. The use of the nitrogen mustards in the palliative treatment of carcinoma. With particular reference to bronchogenic carcinoma. Cancer 1948; 1: 634-56. doi: 10.1002/1097-0142(194811)1:4<634::AIDCNCR2820010410>3.0.CO;2-L

12. Folstein MF, Folstein SE, McHugh PR. Mini-mental state. J Psychiatr Res 1975; 12: 189 98. doi: 10.1016/0022-3956(75)90026-6

13. Lobo A, Ezquerra J, Gómez Burgada F, Sala JM, Seva DïazA. El Miniexamen cognoscitivo (un test sancillo, práctico para detectar alteraciones intelectuales en pacientes medicos). Rev Psiquiatr Psicol Med Eur Am Lat 1980; 14: 39-57.

14. Aaronson NK, Ahmedzai S, Bergman B, Bullinger M, Cull A, Duez NJ, et al. The European Organization for Research and Treatment of Cancer QLQ-C30: a quality-oflife instrument for use in international clinical trials in oncology. J Natl Cancer Inst 1993; 85: $365-76$. 
15. Fayers P, Aaronson N, Bjordal K, Bottomley A, de Graeff A, Groenvold M, et al. EORTC QLQ-C30. Scoring manual. 3.rd Ed. Brussels: EORTC; 2002.

16. Arraras JI, Arias F, Tejedor M, Pruja E, Marcos M, Martínez E, et al. The EORTIC QLQ-C30 (Version 3.0) quality of life questionnaire: Validation study for Spain with head and neck cancer patients. Psychooncology 2002; 11: 249-56. doi: 10.1002/pon.555

17. Bjordal K, de Graeff A, Fayers PM, Hammerlid E, van Pottelsberghe C, Curran D, et al. A 12 country field study of the EORTC QLQ-C30 (version 3.0) and the head and neck cancer specific module (EORTC QLQ-H\&N35) in head and neck patients. EORTC Quality of Life Group. Eur J Cancer 2000; 36: 1796-807. DOI: 10.1016/S0959-8049(00)00186-6

18. Arraras JI, Villafranca E, Vega FA de la, Romero P, Rico M, Vila M, et al. The EORTC Quality of Life Questionnaire for patients with prostate cancer: EORTC QLQ-PR25. Validation study for Spanish patients. Clin Transl Oncol 2009; 11: 160-4. doi 10.1007/ S12094-009-0332-z

19. van Andel G, Bottomley A, Fosså SD, Efficace F, Coens C, Guerif S, et al. An international field study of the EORTC QLQ-PR25: a questionnaire for assessing the health-related quality of life of patients with prostate cancer. Eur J Cancer 2008; 44: 2418-24. doi: 10.1016/j.ejca.2008.07.030

20. Arraras JI, Greimel E, Sezer O, Chie W-C, Bergenmar M, Costantini A, et al. An international validation study of the EORTC QLQ-INFO25 questionnaire: an instrument to assess the information given to cancer patients. Eur J Cancer 2010; 46: 2726-38. doi: 10.1016/j.ejca.2010.06.118.

21. Arraras JI, Manterola A, Hernández B, Arias de la Vega F, Martínez M, Vila M, et al. The EORTC information questionnaire, EORTC QLQ-INFO25. Validation study for Spanish patients. Clin Transl Oncol 2011; 13: 401-10. doi: 10.1007/s12094-011-0674-1.

22. Fosså SD, Bengtsson T, Borre M, Ahlgren G, Rannikko A, Dahl AA. Reduction of quality of life in prostate cancer patients: experience among 6200 men in the Nordic countries. Scand J Urol 2016; 50: 330-7. doi: 10.1080/21681805.2016.1201859.

23. Shinohara N, Maruyama S, Shimizu S, Nishioka K, Abe T, C-Hatanaka K, et al. Longitudinal comparison of quality of life after real-time tumor-tracking intensitymodulated radiation therapy and radical prostatectomy in patients with localized prostate cancer. J Radiat Res 2013; 54: 1095-101. doi: 10.1093/jrr/rrt049.

24. Vanagas G, Mickeviciene A, Ulys A. Does quality of life of prostate cancer patients differ by stage and treatment? Scand J Public Health 2013; 41: 58-64. doi: 10.1177/1403494812467503. Epub 2012 Dec 4.

25. Majumder K, Brandberg Y, Johansson H, Nilsson S, Bergenmar M. Less satisfaction with information in patients with prostate cancer treated with surgery and salvage radiotherapy compared with patients treated with curative radiotherapy alone, despite similar health-related quality of life. Clin Genitourin Cancer 2014; 12: e71-82. doi: 10.1016/j. clgc.2013.11.001.

26. Lamers RED, Cuypers M, Husson O, de Vries M, Kil PJM, Ruud Bosch JLH, et al. Patients are dissatisfied with information provision: perceived information provision and quality of life in prostate cancer patients. Psychooncology 2016; 25: 633-40. doi: 10.1002/pon.3981

27. Davies NJ, Kinman G, Thomas RJ, Bailey T. Information satisfaction in breast and prostate cancer patients: implications for quality of life. Psychooncology 2008; 17:104852. doi: 10.1002/pon.1305

28. Burt J, Caelli K, Moore K, Anderson M. Radical prostatectomy: men's experiences and postoperative needs. J Clin Nurs 2005; 14: 883-90. doi: 10.1111/j.1365-2702.2005.01123.X 